\title{
O impacto do modelo IFLA Library Reference Model na prática catalográfica: casos de uso, vantagens e desvantagens
}

\author{
Rhuan Henrique Alves de Oliveira \\ Bacharelando; Universidade Federal de São Carlos, São Carlos, SP, Brasil \\ rhuan.henrique.oliv@gmail.com; ORCID: https://orcid.org/0000-0001-8348-0350 \\ Fabiano Ferreira de Castro \\ Doutor; Universidade Federal de São Carlos, São Carlos, SP, Brasil \\ fabianocastro@ufscar.br; ORCID: https://orcid.org/0000-0002-8712-2654
}

Ananda Fernanda de Jesus

Doutoranda; Universidade Estadual Paulista, Marília, SP, Brasil

anandafdj@gmail.com; ORCID: https://orcid.org/0000-0001-7873-6040

\begin{abstract}
Resumo: O IFLA Library Reference Model, IFLA LRM, é um modelo conceitual de alto nível que surge da necessidade de consolidação dos três modelos da Família FR, das demandas do contexto tecnológico vigente e da compatibilidade com as melhores práticas do Linked Data. O IFLA LRM trata os dados bibliográficos em sentido amplo, explicitando os princípios gerais que governam a estrutura lógica da informação bibliográfica, atuando como base para formulação de regras de catalogação e implementações de sistemas bibliográficos. Dessa maneira, essa pesquisa tem como objetivo identificar o estado da arte do modelo IFLA LRM, partindo da realização de uma Revisão Sistemática da Literatura, identificando casos de uso, vantagens e desvantagens apontadas pela literatura científica. Foram estabelecidas as seguintes categorias de análise: (i) enfoque dos documentos; (ii) conceito de IFLA LRM; (iii) vantagens e desvantagens do modelo; (iv) casos de uso do IFLA LRM. Como resultado é apresentado o estado da arte sobre o modelo IFLA LRM, bem como as tendências de pesquisa e o impacto do modelo na prática catalográfica. Conclui-se, que o modelo IFLA LRM se apresenta como objeto de investigação de importância capital, uma vez que reflete as mudanças na Catalogação Descritiva, os novos rumos da área no contexto digital, na adequação, no compartilhamento e no reuso dos dados bibliográficos no cenário da Web Semântica e Linked Data.
\end{abstract}

Palavras-chave: Catalogação Descritiva; Modelos conceituais; Dados bibliográficos; IFLA LRM 


\section{Introdução}

Os modelos conceituais, em uma abordagem ampla, são abstrações do mundo real que retratam sua estrutura por meio de conceitos. O modelo Entidade Relacionamento (E-R), como uma metodologia advinda da Ciência da Computação, desenvolvido nas décadas de 70 e 80 , constitui o primeiro passo para o desenvolvimento de um efetivo sistema de processamento de dados, complementado pelos modelos lógico e físico (COYLE, 2016).

Do ponto de vista do universo bibliográfico ${ }^{1}$, os modelos conceituais definem as entidades, os atributos e os relacionamentos, tendo como objetivo fornecer uma estrutura consistente que contemple requisitos funcionais para os dados bibliográficos.

Segundo Simionato (2017, p. 2-3), esses modelos:

[...] acarretam em uma desconstrução do registro em que os dados de uma estrutura monolítica passam a ser retratados por relações, isto significa que a estrutura fixa de preenchimento de campos e atributos modifica-se para relacionamento entre entidades e atributos.

É a intersecção dessas duas abordagens que compõe o primeiro modelo da Família FR e do universo bibliográfico, o Functional Requirements for Bibliographic Records (FRBR), publicado em 1998. Posteriormente, foram desenvolvidos os modelos Functional Requirements for Authority Data (FRAD), em 2009 e o Functional Requirements for Subject Authority Data (FRSAD), em 2010, buscando atender demandas específicas do universo bibliográfico, respectivamente, dados de autoridade e de assunto.

"O modelo de dados bibliográficos FRBR é a mudança mais radical para o pensamento catalográfico de bibliotecas desde que Panizzi desenvolveu suas 91 regras para o Catálogo do Museu Britânico". (COYLE, 2016, p. 65, tradução nossa). E não somente o FRBR, mas os dois modelos posteriores também, de tal maneira que a Declaração dos Princípios Internacionais de Catalogação (PIC) de 2016, "se constrói sobre a base das grandes tradições catalográficas do mundo e também sobre os modelos conceituais da família dos Requisitos Funcionais da IFLA”. (IFLA, 2016, p. 4). 
Os três modelos da Família FR partem de pontos de vista diferentes. O FRBR tem como enfoque os registros bibliográficos, o FRAD os dados de autoridade e o FRSAD os dados de assunto. Isso tem por consequência, propostas de resoluções diferentes para os mesmos problemas, estabelecendo a demanda pela consolidação desses instrumentos em um único modelo.

O IFLA Library Reference Model (IFLA LRM) é um modelo conceitual de alto nível ${ }^{2}$, estruturado a partir do modelo Entidade-Relacionamento Estendido (EER), que consolida os três modelos da Família FR: FRBR, FRAD e FRSAD. Tratando os dados bibliográficos em sentido amplo, o modelo explicita princípios gerais que governam a estrutura lógica da informação bibliográfica, visando servir de guia ou base para a formulação de regras de catalogação e implementação de sistemas bibliográficos. (RIVA; LEBOEUF; ŽUMER, 2017).

Por ter modelagem EER, o modelo ganhou uma abordagem e uma série de conceitos novos em relação à Família FR, modulada por sua vez em Entidade Relacionamento (ER). Sendo assim, as Entidades no IFLA LRM são tratadas como Classes e como tais configuram-se em Superclasses ou Subclasses, apresentando uma estrutura hierárquica.

Considerando o contexto apresentado, a questão norteadora da pesquisa consiste em verificar como o modelo conceitual IFLA LRM tem sido discutido nas publicações científicas internacionais, a partir da análise de casos de uso, das vantagens e das desvantagens apontadas pela corrente de autores.

Sendo o resultado do processo de consolidação dos três modelos conceituais que compõem a Família FR, cujo impacto na prática da catalogação descritiva é iminente, essa pesquisa visa atender demandas do ambiente tecnológico vigente, e parte do objetivo de apresentar o estado da arte do IFLA LRM na literatura científica disponível e o cenário de desenvolvimento do modelo, na perspectiva da pesquisa e de sua aplicação no campo da Catalogação Descritiva. 


\section{Percurso metodológico}

De caráter exploratório e descritivo, essa pesquisa adota como método, a Revisão Sistemática da Literatura (RSL).

A Revisão Sistemática da Literatura é um método de pesquisa composto de um corpo estruturado de protocolos, de etapas e de critérios prédefinidos, que garantem alta e comprovável qualidade, pois é

[...] focada no seu caráter de reprodutibilidade por outros pesquisadores, apresentando de forma explícita as bases de dados bibliográficos que foram consultadas, as estratégias de busca empregadas em cada base, o processo de seleção dos artigos científicos, os critérios de inclusão e exclusão dos artigos e o processo de análise de cada artigo. Explicita ainda as limitações de cada artigo analisado, bem como as limitações da própria revisão. (GALVÃO; RICARTE, 2019, p. 58-59).

Nessa pesquisa, a Revisão Sistemática da Literatura foi conduzida em três etapas principais, conforme apresentado na Figura 1.

Figura 1 - Etapas da Revisão Sistemática da Literatura.

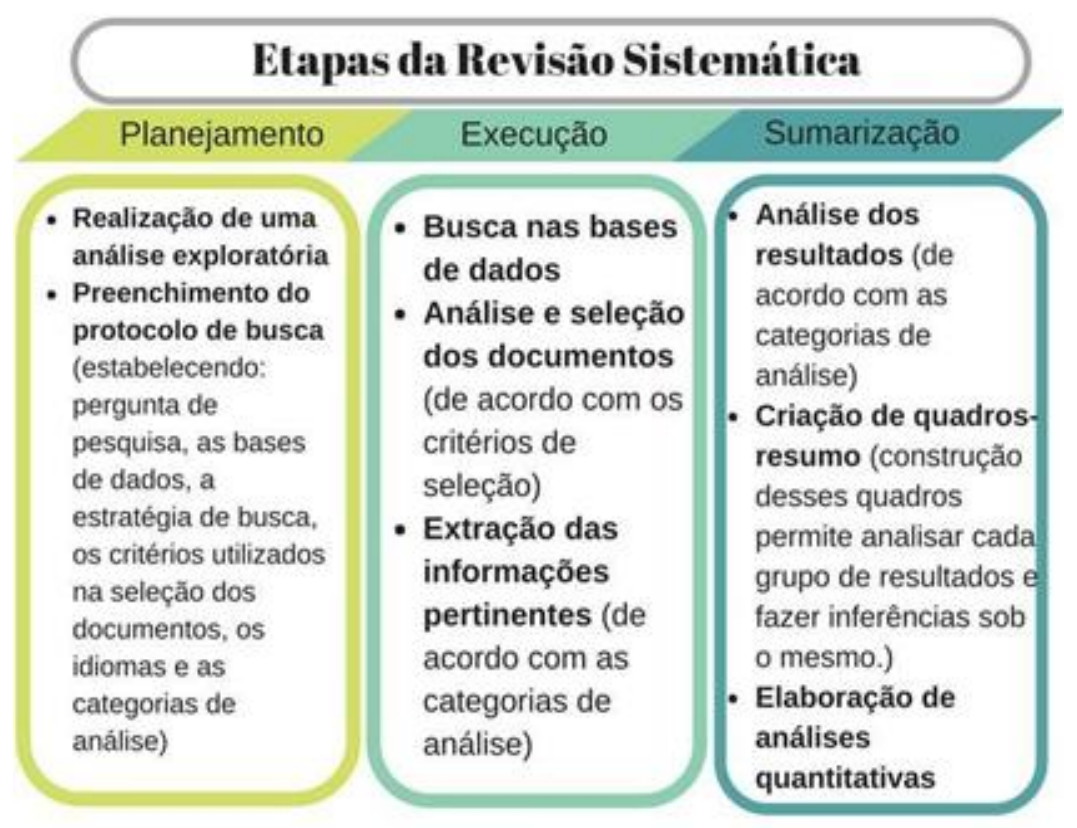

Fonte: Jesus e Castro (2019, p. 47). 
Para auxiliar no processo de realização, utilizou-se a ferramenta denominada State of the Art through Systematic Review (StArt), desenvolvida pelo Laboratório de Pesquisa em Engenharia de Software (LAPES), do Departamento de Ciência da Computação, da Universidade Federal de São Carlos (UFSCar). O StArt conta com um protocolo no qual são registradas informações importantes, como palavras-chave, critérios de inclusão e de exclusão, bases de dados consultadas, categorias de análise etc., como visualizado no Quadro 1.

Quadro 1 - Protocolo de Busca da RSL

\begin{tabular}{|c|c|}
\hline & Protocolo da Revisão Sistemática \\
\hline Objetivos & $\begin{array}{l}\text { Identificar e apresentar o estado da arte do modelo IFLA LRM } \\
\text { na literatura científica disponível e seus casos de uso. }\end{array}$ \\
\hline Questão principal & $\begin{array}{l}\text { Como o modelo conceitual IFLA LRM tem sido discutido nas } \\
\text { publicações científicas? }\end{array}$ \\
\hline População & $\begin{array}{l}\text { Resultados de projetos e casos de uso do modelo que permitam } \\
\text { desenvolver um corpus teórico. }\end{array}$ \\
\hline Intervenção & $\begin{array}{l}\text { Estudos conceituais ou práticos do modelo conceitual IFLA } \\
\text { LRM. }\end{array}$ \\
\hline Controle & $\begin{array}{l}\text { Análise exploratória do tema, considerando artigos científicos } \\
\text { publicados sobre a temática. }\end{array}$ \\
\hline Resultados & $\begin{array}{l}\text { Deseja-se a construção de uma base teórica consolidada acerca } \\
\text { do modelo conceitual IFLA LRM, a qual permita identificar a } \\
\text { maneira que o modelo vem sendo aplicado na prática. }\end{array}$ \\
\hline Aplicação & $\begin{array}{l}\text { Pesquisadores da Ciência da Informação que trabalhem com } \\
\text { Catalogação Descritiva e bibliotecários. }\end{array}$ \\
\hline $\begin{array}{l}\text { Palavras-chave e } \\
\text { Sinônimos }\end{array}$ & $\begin{array}{c}\text { FRBR Family. Família FR. IFLA LRM. IFLA Library } \\
\text { Reference Model. FRBR LRM. FRBR-Library Reference } \\
\text { Model. Modelos conceituais. Concept models. Modelos } \\
\text { conceptuales. Catalogação descritiva. Descriptive Cataloging. } \\
\text { Catalogación descritiva. }\end{array}$ \\
\hline
\end{tabular}




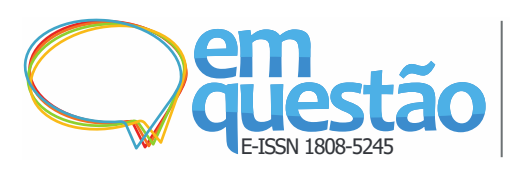

O impacto do modelo IFLA Library Reference Model na prática catalográfica: casos de uso, vantagens e

desvantagens

Rhuan Henrique Alves de Oliveira, Fabiano Ferreira de Castro,

Ananda Fernanda de Jesus

\begin{tabular}{|c|c|}
\hline Bases de dados & $\begin{array}{c}\text { Web of Science; Library and Information Science Abstracts } \\
\text { (LISA); Information Science \& Technology Abstracts (ISTA); } \\
\text { Library, Information Science \& Technology Abstracts with full } \\
\text { text (LISTA) e Base de dados de Periódicos em Ciência da } \\
\text { Informação (BRAPCI). }\end{array}$ \\
\hline $\begin{array}{c}\text { Definição dos } \\
\text { Critérios de seleção } \\
\text { de fontes }\end{array}$ & $\begin{array}{l}\text { Artigos científicos publicados em periódicos da área de } \\
\text { Biblioteconomia e Ciência da Informação. }\end{array}$ \\
\hline $\begin{array}{l}\text { Linguagem dos } \\
\text { estudos }\end{array}$ & Português. Inglês. Espanhol. \\
\hline $\begin{array}{c}\text { Métodos de } \\
\text { indagação das fontes }\end{array}$ & $\begin{array}{c}\text { Leitura do título e resumo dos documentos, aplicação dos } \\
\text { critérios de inclusão e de exclusão, leitura da introdução e da } \\
\text { conclusão, ou ainda do documento completo, nos casos em que } \\
\text { as análises anteriores se mostrarem inconclusivas para a } \\
\text { seleção. }\end{array}$ \\
\hline $\begin{array}{c}\text { Critério de seleção } \\
\text { de estudos (inclusão } \\
\text { e exclusão) }\end{array}$ & $\begin{array}{l}\text { (I) Trabalhos que discutem os termos estabelecidos. } \\
\text { (I) Trabalhos que discutem casos de uso do modelo IFLA LRM } \\
\text { (I) Trabalhos que discutem os conceitos estabelecidos. } \\
\text { (E) Trabalhos que mencionam os termos apenas no resumo ou } \\
\text { apenas no texto sem conceituá-los ou discuti-los. } \\
\text { (E) Trabalhos que mencionam os termos apenas nas palavras- } \\
\text { chave. } \\
\text { (E) Trabalhos que mencionam os termos apenas em tabelas e } \\
\text { quadros. } \\
\text { (E) Trabalhos que mencionam os termos apenas nas } \\
\text { referências. } \\
\text { (E) Trabalhos que mencionam os termos apenas em notas de } \\
\text { rodapé. } \\
\text { (E) Trabalhos em línguas fora das pré-estabelecidas no } \\
\text { protocolo. }\end{array}$ \\
\hline $\begin{array}{l}\text { Definição dos tipos } \\
\text { de estudos }\end{array}$ & $\begin{array}{l}\text { Artigos publicados em periódicos científicos, sem restrição do } \\
\text { período de publicação. }\end{array}$ \\
\hline $\begin{array}{l}\text { Seleção inicial de } \\
\text { estudos }\end{array}$ & Não se aplica. \\
\hline $\begin{array}{l}\text { Critério de seleção } \\
\text { de fontes }\end{array}$ & $\begin{array}{l}\text { Os documentos serão avaliados seguindo os critérios de } \\
\text { cientificidade dos periódicos. }\end{array}$ \\
\hline $\begin{array}{l}\text { Campos para } \\
\text { avaliação da } \\
\text { qualidade }\end{array}$ & $\begin{array}{l}\text { - Metodologia do Estudo; } \\
\text { - Método de avaliação do periódico. }\end{array}$ \\
\hline
\end{tabular}




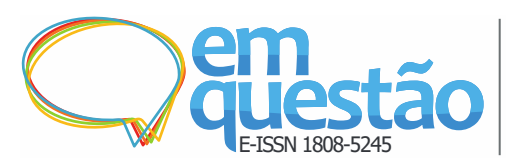

O impacto do modelo IFLA Library Reference Model na prática catalográfica: casos de uso, vantagens e

desvantagens

Rhuan Henrique Alves de Oliveira, Fabiano Ferreira de Castro, Ananda Fernanda de Jesus

\begin{tabular}{|c|c|}
\hline $\begin{array}{c}\text { Campo para } \\
\text { extração dos dados }\end{array}$ & $\begin{array}{l}\text { - Enfoque do documento } \\
\text { - Conceito de IFLA Library Reference Model } \\
\text { - Relação entre o modelo IFLA Library Reference Model } \\
\text { com o Linked Data } \\
\text { - Vantagens do modelo } \\
\text { - Desvantagens do modelo } \\
\text { - Casos de uso do modelo IFLA LRM }\end{array}$ \\
\hline $\begin{array}{l}\text { Sumarização dos } \\
\text { resultados }\end{array}$ & $\begin{array}{c}\text { Agrupar os resultados em quadros de acordo com a categoria } \\
\text { de análise, permitindo a realização de análises quantitativa e } \\
\text { qualitativa dos conceitos estabelecidos. }\end{array}$ \\
\hline
\end{tabular}

Fonte: Elaborado pelos autores.

As buscas nas bases de dados selecionadas e apontadas no protocolo da Revisão Sistemática da Literatura foram realizadas primeiramente em 26 de setembro de 2019 e, posteriormente, atualizadas em 26 de maio de 2020 e em 22 de janeiro de 2021.

A estratégia de busca foi definida a partir de variações do nome do modelo, que até 2016 foi FRBR-LRM e a partir de 2017 tornou-se IFLA LRM; dessa maneira, seguiu-se a elaboração da string de busca: ("IFLA LRM" OR "IFLA Library Reference Model” OR "FRBR LRM" OR "FRBR-Library Reference Model"). Optou-se por uma estratégia de busca mais geral pautada no caráter recente do modelo analisado, partindo da hipótese de que haveria número limitado de artigos, fazendo-se necessária uma abordagem mais abrangente na composição da estratégia da busca.

\section{Análise dos resultados e discussão}

As bases utilizadas foram Web Of Science, Library and Information Science Abstracts (LISA), Library, Information Science \& Technology Abstracts with full text (LISTA), Information Science \& Technology Abstracts (ISTA) e Base de dados de Periódicos em Ciência da Informação (BRAPCI), das quais foram recuperados um total de 168 resultados, como visto na Figura 2. 
Figura 2 - Resultados recuperados e suas respectivas bases de dados.

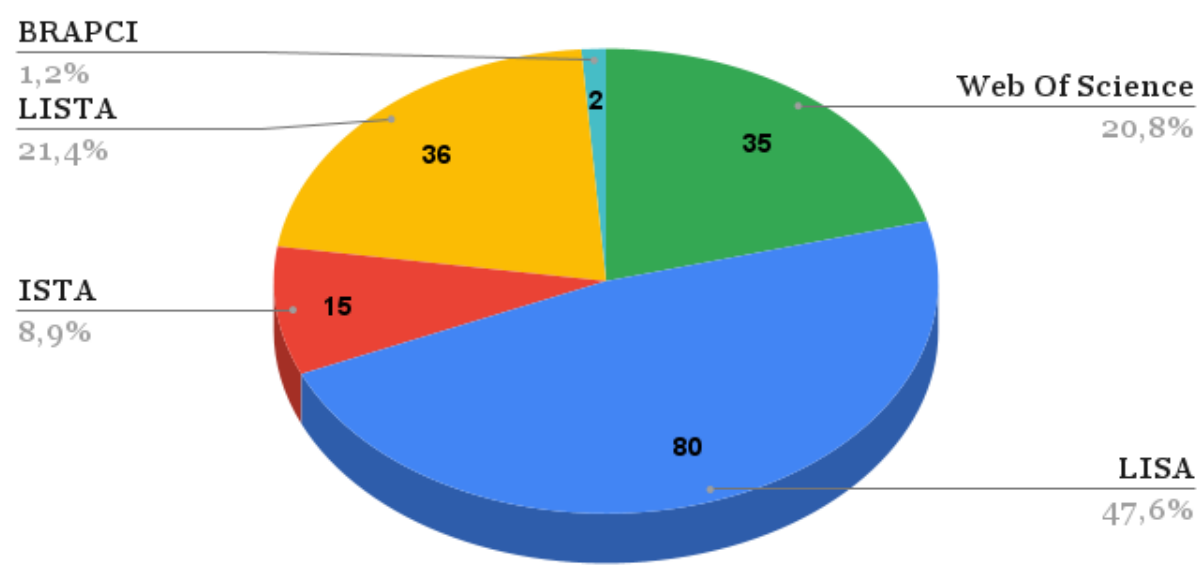

Fonte: Dados da pesquisa.

É possível notar a predominância dos resultados recuperados na LISA e o baixo alcance dos resultados da BRAPCI sobre o assunto, o que é justificado por se tratar de uma iniciativa recente e desenvolvida no âmbito internacional, o que pressupõe a necessidade de estudos mais aprofundados no cenário brasileiro.

Após a leitura do título, do resumo e das palavras-chave dos documentos, realizou-se a aplicação dos critérios de inclusão e de exclusão, conforme a Figura 3. 
Figura 3 - Fase de extração dos documentos.

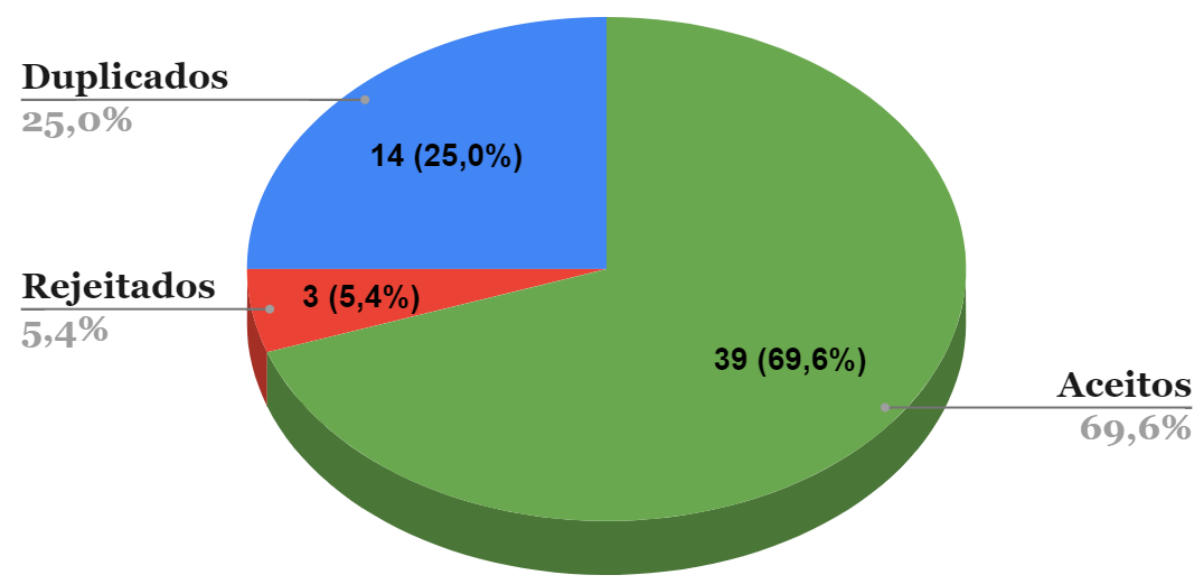

Fonte: Dados da pesquisa.

Dos 168 documentos recuperados, foram selecionados 39 artigos, sendo 14 artigos duplicados e 3 artigos rejeitados. Desse modo, a massa documental dessa pesquisa é constituída por 39 documentos que abordam o modelo IFLA LRM, que foram analisados conforme a discussão do IFLA LRM e seus casos de uso, vantagens e desvantagens.

\subsection{Casos de uso}

A partir da análise dos documentos aceitos, foram identificados seis casos de uso do modelo IFLA LRM, esse número reduzido de casos pode, em partes, ser justificado pela publicação recente do modelo, em 2017.

Quadro 2 - Casos de uso do modelo IFLA LRM.

\begin{tabular}{|c|c|c|l|}
\hline Autor(res) & \multicolumn{1}{|c|}{ Título } & Ano & \multicolumn{1}{c|}{ Caso de Uso } \\
\hline AALBERG, Trond & $\begin{array}{c}\text { The impact of new } \\
\text { TALLERÅS, Kim } \\
\text { MASSEY, David } \\
\text { the search experience }\end{array}$ & \multirow{2}{*}{2019} & $\begin{array}{l}\text { Conversão de registros LRM em } \\
\text { dados RDF de acordo com o } \\
\text { BIBFRAME e o IFLA LRM, } \\
\text { implementando ambos os modelos } \\
\text { em um protótipo de busca. }\end{array}$ \\
\hline
\end{tabular}


Rhuan Henrique Alves de Oliveira, Fabiano Ferreira de Castro, Ananda Fernanda de Jesus

\begin{tabular}{|c|c|c|c|}
\hline $\begin{array}{l}\text { ARASTOOPOOR, } \\
\text { Sholeh }\end{array}$ & $\begin{array}{c}\text { Users' perception of } \\
\text { navigating bibliographic } \\
\text { families from IFLA-LRM } \\
\text { perspective }\end{array}$ & 2020 & $\begin{array}{l}\text { Baseando-se no IFLA LRM, análise } \\
\text { da percepção de famílias } \\
\text { bibliográficas por parte dos usuários; } \\
\text { tanto em uma situação em que não há } \\
\text { documento específico em mente, } \\
\text { quanto em outra que há situação de } \\
\text { necessidade pré-definida. Dessa } \\
\text { maneira busca-se verificar se há um } \\
\text { padrão de como os usuários veem as } \\
\text { famílias bibliográficas. }\end{array}$ \\
\hline BENELLI, Sabina & $\begin{array}{c}\text { Italian Cataloguing Rules } \\
\text { For Unpublished Music } \\
\text { Resources }\end{array}$ & 2020 & $\begin{array}{l}\text { Regras de catalogação para recursos } \\
\text { musicais não publicados baseadas na } \\
\text { análise do IFLA LRM e outros } \\
\text { modelos conceituais. }\end{array}$ \\
\hline $\begin{array}{c}\text { MARCONDES, C. } \\
\text { H. }\end{array}$ & $\begin{array}{l}\text { Towards a vocabulary to } \\
\text { implement culturally } \\
\text { relevant relationships } \\
\text { between digital collections } \\
\text { in heritage institutions }\end{array}$ & 2020 & $\begin{array}{l}\text { Utilização do IFLA LRM como fonte } \\
\text { para identificação de possíveis } \\
\text { relacionamentos culturalmente } \\
\text { relevantes entre objetos. }\end{array}$ \\
\hline $\begin{array}{c}\text { PADRON, Marcos } \\
\text { Fragomeni } \\
\text { CRUZ, Fernando } \\
\text { William } \\
\text { SILVA, Juliana } \\
\text { Rocha de Faria }\end{array}$ & $\begin{array}{c}\text { Extending the IFLA } \\
\text { Library Reference Model } \\
\text { for a Brazilian popular } \\
\text { music digital library }\end{array}$ & 2020 & $\begin{array}{l}\text { Experimentação do mecanismo de } \\
\text { extensão do IFLA LRM aplicando-o } \\
\text { em uma biblioteca digital de música } \\
\text { popular brasileira. }\end{array}$ \\
\hline $\begin{array}{l}\text { TANIGUCHI, } \\
\text { Shoici }\end{array}$ & $\begin{array}{l}\text { Mapping and merging of } \\
\text { IFLA Library Reference } \\
\text { Model and BIBFRAME } \\
2.0\end{array}$ & 2018 & $\begin{array}{l}\text { Mapeamento e a fusão entre o IFLA } \\
\text { LRM e o BIBFRAME 2.0. }\end{array}$ \\
\hline
\end{tabular}

Fonte: Elaborado pelos autores.

No primeiro caso, inserido no contexto da experiência de busca bibliográfica do usuário, Aalberg, Tallerås e Massey (2019) converteram registros MARC em dados Resource Description Framework (RDF) de acordo com o BIBFRAME e $o$ LRM, implementando ambos os modelos em um protótipo de busca. Na conceituação,

[...] os dois modelos facilitam a representação de informação bibliográfica, porém utilizando diferentes estruturas que também criam diferenças significativas nas diferentes maneiras como o usuário pode interagir e explorar os dados (AALBERG; TALLERÅS; MASSEY, 2019, n.p., tradução nossa). 
Como resultados desse estudo, Aalberg, Tallerås e Massey (2019, tradução nossa) apontam que o IFLA LRM, em comparação com o BIBFRAME, provê uma lista de resultados mais curtas, com estrutura mais complexa e articulada, de maneira a habilitar acesso às manifestações.

O LRM permite que manifestações incorporem múltiplas expressões, e sempre haverá uma trajetória da Obra para a Manifestação direta e consistente para qualquer fração do conteúdo que seja catalogado, o que implica que para qualquer Obra encontraremos todas as Manifestações que incorporam uma Expressão da Obra (AALBERG; TALLERÅS; MASSEY, 2019, n.p., tradução nossa).

Desse modo, apesar da estrutura semelhante, no que diz respeito às entidades dos modelos BIBFRAME e IFLA LRM, o primeiro não dispõe da consistência e simplicidade do segundo, no agrupamento de produtos intelectuais (AALBERG; TALLER ÅS; MASSEY, 2019, tradução nossa).

Buscando entender a percepção que os usuários têm das famílias bibliográficas e como navegam através delas, Arastoopoor (2020) utilizou fichas catalográficas (para card-sorting), entrevistas semiestruturadas e questionários, avaliando as situações com base no IFLA LRM. A partir disso, o estudo buscou responder duas questões: como os usuários de OPACs concebem amplas famílias bibliográficas quando não possuem um documento específico da família em mente, e quando possuem a intenção de recuperar um documento específico participante dessa família.

A obra escolhida para o estudo foi o poema épico persa "Epic of Kings", devido à sua ampla família bibliográfica e, como participantes (que não foram diretamente expostos às entidades do modelo) foram selecionados estudantes de doutorado e doutores que possuem formação em Literatura Persa, e que produziram estudos sobre a obra. Um conjunto de 40 fichas bibliográficas que representam a família da obra foi preparado para o card-sorting, cada uma dessas fichas correspondendo a entidades $\mathrm{OEMI}^{3}$. Dessa maneira, os dados foram coletados a partir do card-sorting, de entrevistas posteriores que buscaram entender o porquê das tomadas de decisões dos usuários no card- 
sorting e das questões complementares elaboradas no formato de uma entrevista semiestruturada (ARASTOOPOOR, 2020).

Descobriu-se a partir dos dados coletados que os usuários utilizam a estrutura dos relacionamentos hierárquicos, que possuem uma percepção acurada da entidade Res em seu fator agregador e, consequentemente, que os relacionamentos mais identificados por parte deles são o LRM-R1 e LRM-R12.

A entidade Res (“coisa" em latim), inédita do LRM, é definida como

[...] a entidade superior do modelo. Res inclui coisas e conceitos materiais ou físicos. Tudo o que é considerado relevante para o universo bibliográfico, que é o universo do discurso neste caso, está incluído. Res é uma superclasse de todas as outras entidades explicitamente definidas, bem como de quaisquer outras entidades não especificamente identificadas (RIVA; LEBOEUF; ŽUMER, 2017, p. 21).

Em outras palavras, todas as outras entidades são subclasses da entidade Res, ou seja, agrupadas por ela, e consequentemente herdam todos seus atributos e relacionamentos. Dessa maneira, Obra, Expressão, Manifestação e Item são entidades desmembradas, porém possuem a mesma relação com a entidade superior Res, o de ser subclasse, e é isso fica evidente para os usuários (sem conhecimento prévio do modelo, de suas entidades etc.) em suas percepções da família bibliográfica, como aponta Arastoopoor (2020).

O LRM-Rl tem, tanto como domínio, quanto como abrangência, a entidade Res, e indica uma associação entre duas Res. Já o LRM-R12 é um relacionamento que tem como domínio a entidade Obra, e como abrangência a entidade Res, indicando que a primeira possui como assunto a segunda (RIVA; LEBOEUF; ŽUMER, 2017).

Benelli (2020), por sua vez, relata o processo de elaboração das Italian Cataloguing Rules for Unpublished Music Resources, aplicadas a manuscritos musicais, partituras, áudios e gravações não publicadas e outros recursos relacionados. Essas regras de catalogação basearam-se na análise do IFLA LRM e outros modelos conceituais e regras de catalogação. 
As contribuições do IFLA LRM para o desenvolvimento dessas regras são concernentes principalmente ao conceito de manifestação unitária, que aplicado aos recursos musicais não publicados contribui para a "identificação correta dos níveis de manifestação e item na descrição bibliográfica de tais recursos", pois

De acordo com o IFLA LRM, no caso de recursos não publicados, todos os elementos concebidos durante o processo de produção pertencem à sua manifestação (projetado como nível da manifestação), enquanto todas as modificações posteriores pertencem ao item (BENELLI, 2020, p. 322, tradução nossa).

Tal conceituação é extraída das notas de escopo da entidade manifestação do IFLA LRM, que postula o seguinte:

Em outros casos, como para manuscritos holográficos, muitas produções ou reproduções artesanais ou artísticas para fins de preservação, a intenção é que o processo de produção resulte em um único item. A manifestação, nesse caso, é o conjunto unitário (um conjunto com um único componente) que captura a ideia do item em questão (RIVA; LEBOEUF; ŽUMER, 2017, p. 26).

Marcondes (2020) em seu artigo Towards a Vocabulary to Implement Culturally Relevant Relationships Between Digital Collections in Heritage Institutions, examina o IFLA LRM como fonte de consulta para identificação de possíveis relacionamentos culturalmente relevantes entre objetos. Dessa maneira, o autor destaca os relacionamentos prescritos na tabela 4.7 do documento final do IFLA LRM, que contém todos os relacionamentos possíveis entre entidades prescritos pelo modelo, junto com seus domínios, abrangência e cardinalidade (RIVA; LEBOEUF; ŽUMER, 2017).

Dentre um desses relacionamentos culturalmente relevantes analisados por Marcondes (2020), o "inspired relationship", o autor encontra paralelo no relacionamento LRM- $R 21^{4}$, do IFLA LRM. Esse relacionamento no vocabulário desenvolvido no artigo é descrito como: "relacionamento entre um livro que inspirou uma pintura ou desenho, entre uma obra de arte e um livro. Relacionamentos entre duas obras independentes com diferentes autores". O relacionamento do LRM, por sua vez, é definido como: "relacionamento entre 
duas obras em que o conteúdo da primeira serviu como fonte de ideias para o segundo" (RIVA; LEBOEUF; ŽUMER, 2017).

Padron, Cruz e Silva (2020), buscaram experimentar o mecanismo de extensão do modelo aplicando-o em uma biblioteca digital de música popular brasileira. Para essa aplicação foram seguidas as postulações do documento final de que, o modelo deve ser usado como base ou guia para a implementação de sistemas bibliográficos, porém “[...] sendo necessário reter os relacionamentos entre as entidades OEMI para uma aplicação ser considerada implementação do modelo" (RIVA; LEBOEUF; ŽUMER, 2017, p. 10).

Esse caso pautou-se na aplicação da estrutura do IFLA LRM em um modelo pré-existente de representação da Música Popular brasileira, que identifica criações musicais, melodias das composições e seus arranjos, além de suas relações. (PADRON; CRUZ; SILVA, 2020). Para tal procedimento foram utilizados os mecanismos de extensão previstos pelo IFLA LRM.

\begin{abstract}
Estender o modelo IFLA LRM é uma tarefa de modelagem conceitual que implica (i) a identificação e definição dos conceitos que são específicos para o domínio da aplicação, (ii) avaliação de aplicação de conceitos bibliográficos existentes e (iii) compreensão de como esses conceitos (bibliográficos e específicos da aplicação) relacionam-se entre si (PADRON; CRUZ; SILVA, 2020, p. 14, tradução nossa).
\end{abstract}

No que concerne aos mecanismos de extensão (ou expansão, conforme a tradução para o português do documento do modelo), é postulado que:

O IFLA LRM fornece vários mecanismos que permitem as expansões que provavelmente serão necessárias em qualquer implementação real. A definição do atributo categoria para a entidade res permite que implementações criem, para qualquer uma das entidades, as subclasses que podem ser úteis. Atributos especializados adicionais podem ser acrescentados a uma ou a todas as entidades, seguindo os padrões fornecidos, para abranger, por exemplo, tipos de recursos específicos ou fornecer mais detalhes sobre agentes (RIVA; LEBOEUF; ŽUMER, 2017, p. 10).

Constata-se a utilização desses mecanismos por parte de Padron, Cruz e Silva (2020) na criação de quatro novas entidades (MPB-E1 a MPB-E4) como subclasses de Res. Essas entidades correspondem a circunstâncias específicas do 
domínio musical, sendo elas Gênero Musical, Notação Musical, Performance Individual e Performance em Banda, atendendo dessa maneira, as necessidades do caso de uso em voga.

Como conclusão do estudo de Padron, Cruz e Silva (2020, p. 16, tradução nossa), foi demonstrado que a aplicação específica produziu um modelo bibliográfico "alinhado com a visão do usuário, com metadados enriquecidos e capaz de responder complexas questões de busca que catálogos tradicionais não conseguiriam".

Taniguchi (2018) propõe o mapeamento e a fusão entre o IFLA LRM e o BIBFRAME 2.0. O autor conceitua o mapeamento de vocabulário de metadados como subárea e o alinhamento de ontologias e seu objetivo, como julgar e determinar correspondências entre termos de diferentes vocabulários. A fusão de vocabulário de metadados, por sua vez, é uma tarefa que vai além do mapeamento lidando de maneira mais minuciosa com equivalência de relacionamentos, hierarquias e outros dos diferentes vocabulários.

Em princípio, Taniguchi (2018) converteu a modelagem EntidadeRelacionamento Estendido do IFLA LRM para o RDF, já utilizado pelo BIBFRAME como linguagem de modelagem. As entidades do IFLA LRM foram convertidas em classes RDF sem nenhuma modificação. Os atributos e os relacionamentos do modelo foram ambos convertidos para propriedades RDF; a cardinalidade prescrita pelo IFLA LRM não foi incorporada (por mais que ela possa ser representada via Web Ontology Language $\left(\mathrm{OWL}^{5}\right)$, o BIBFRAME não a possui), assim como as tarefas do usuário não foram referidas devido à sua impossibilidade de expressão em RDF ou OWL (TANIGUCHI, 2018).

Em suma, o estudo demonstrou três principais diferenças entre os modelos:

[...] (a) maneiras de desenvolvimento das classes essenciais para representação de recursos bibliográficos, agentes, nomens etc.; (b) aquelas que representam a responsabilidade do agente pelos recursos bibliográficos, tanto com classes de evento ou sem elas; e (c) aquelas que especificam o domínio das propriedades tanto em uma perspectiva restritiva ou em uma flexível. (TANIGUCHI, 2018, p. 453 , tradução nossa). 
A partir desse estudo de Taniguchi (2018), é possível inferir alguns rumos que a catalogação descritiva está tomando e, concordando com Sprochi (2016), alinhar o IFLA LRM, juntamente com o BIBFRAME e o Resource Description and Access (RDA), como as três iniciativas com maior potencial de transformação na prática da catalogação, e que suas adoções hão de resultar em grandes mudanças no domínio bibliográfico, além de estarem profundamente entrelaçadas e dependerem uma das outras para serem implementadas.

Dessa maneira, verifica-se que os casos de uso apresentam resultados muito positivos para o IFLA LRM, justificando dessa maneira algumas diretrizes de sua elaboração, principalmente seu nível amplo de abstração.

\subsection{Vantagens e desvantagens do modelo IFLA LRM}

No que concerne às vantagens e às desvantagens do modelo IFLA LRM, de acordo com os resultados da RSL, em quinze artigos constam fatores favoráveis ao desenvolvimento e, em treze artigos, fatores desfavoráveis ao modelo, sendo que muitas vezes é o mesmo documento que os contrapõe. Neste balanço, não foram levados em conta os artigos de casos de uso que apresentaram resultados positivos, porém nota-se que alguns desses resultados refutam, no campo prático, como supracitado, algumas desvantagens apontadas por outros artigos no campo conceitual.

Strader (2017, p. 355, tradução nossa) aponta que a diferença entre os três modelos FR tinha razão de ser, afinal: "dados de autoridade não são a mesma coisa que dados bibliográficos". Portanto, como consequência da consolidação ocorrida no IFLA LRM, o autor destaca - sendo citado também no artigo de Pennington e Cagnazzo (2018) -, que:

Como a intenção do FBRB-LRM é harmonizar os três modelos em um único modelo "orientado para o exterior", é postulado aqui que há necessariamente uma perda de granularidade, assim como de algum poder funcional e explanatório, como resultado dessa consolidação. Além disso, essa perda de granularidade enfraquece o modelo exatamente no lugar onde a infraestrutura necessita ser reforçada para máximo suporte da última tarefa do usuário, especialmente para a criação de pontos de acesso que permitem 
relacionamentos bibliográficos vir à tona (STRADER, 2017, p. 354355 , tradução nossa).

Dunsire, Fritz e Fritz (2019), discorrendo sobre o cenário atual da Catalogação descritiva, elogiam a introdução da entidade Nomen no IFLA LRM, pois ela permite uma distinção clara entre as etiquetas/termos (nomes, títulos, identificadores etc.) e as coisas em si (representadas pela entidade Res). Dessa maneira, os autores destacam que é possível ver nessa distinção, uma adaptação de termos familiares a novos contextos, uma vez que a prática catalográfica adentrou as fronteiras da Web Semântica e Linked Data.

Ademais, Frederick (2019), em seu artigo Information seeking in the age of data deluge, aponta que com a revisão das tarefas do usuário, o modelo consegue abranger tarefas que seriam realizadas em casos de busca de informação em qualquer cenário e plataforma, de tal maneira que o modelo pode ser usado para estudos de como os seres humanos buscam informação em um contexto geral.

As tarefas do usuário, apresentadas no Quadro 3, definem o escopo funcional do modelo. Em relação ao FRBR, quatro dessas tarefas já se encontravam presentes, a quinta e a última, porém é inédita do FRSAD e foi mantida no IFLA LRM. Hider (2016) chama a atenção ao fato de que, já nos anos de 2000, Svenonius fez a proposta para o FRBR com o nome de "navegar".

Quadro 3 - Sumário das tarefas dos usuários.

\begin{tabular}{|c|l|}
\hline Encontrar & $\begin{array}{l}\text { Agrupar informação sobre um ou mais recursos de interesse } \\
\text { através da busca por qualquer critério relevante. }\end{array}$ \\
\hline Identificar & $\begin{array}{l}\text { Compreender claramente a natureza dos recursos encontrados e } \\
\text { distinguir entre recursos similares. }\end{array}$ \\
\hline Selecionar & $\begin{array}{l}\text { Determinar a utilidade dos recursos encontrados, e ser capaz de } \\
\text { aceitar ou rejeitar recursos específicos. }\end{array}$ \\
\hline Obter & Acessar o conteúdo do recurso. \\
\hline Explorar & $\begin{array}{l}\text { Descobrir recursos utilizando-se da relação entre eles e então } \\
\text { enquadrá-los em um contexto. }\end{array}$ \\
\hline
\end{tabular}

Fonte: Riva, LeBouef e Žumer (2017, p. 15). 
No âmbito das publicações seriadas, Jones (2018, p. 128, tradução nossa), mostra que "[...] o LRM abandona a estrutura padrão do FRBR, onde uma obra pode ser realizada em múltiplas expressões e incorporada em múltiplas manifestações”. Dessa maneira, o IFLA LRM:

[...] modela o mundo real, de tal maneira que sugere uma estrutura do Grupo 1 do FRBR achatada para publicações seriadas, onde todos os relacionamentos ocorrem efetivamente no nível da Manifestação e cada Manifestação possui uma relação um-para-um com a Expressão e a Obra que ela incorpora [...] De acordo com o IFLA LRM, duas manifestações seriadas sempre vão incorporar diferentes Expressões e Obras. (JONES, 2018, p. 142, tradução nossa).

Dessa forma, a Figura 4 retrata a modelagem de agregados prescrita pelo modelo IFLA LRM.

Figura 4 - Modelo geral para agregados.

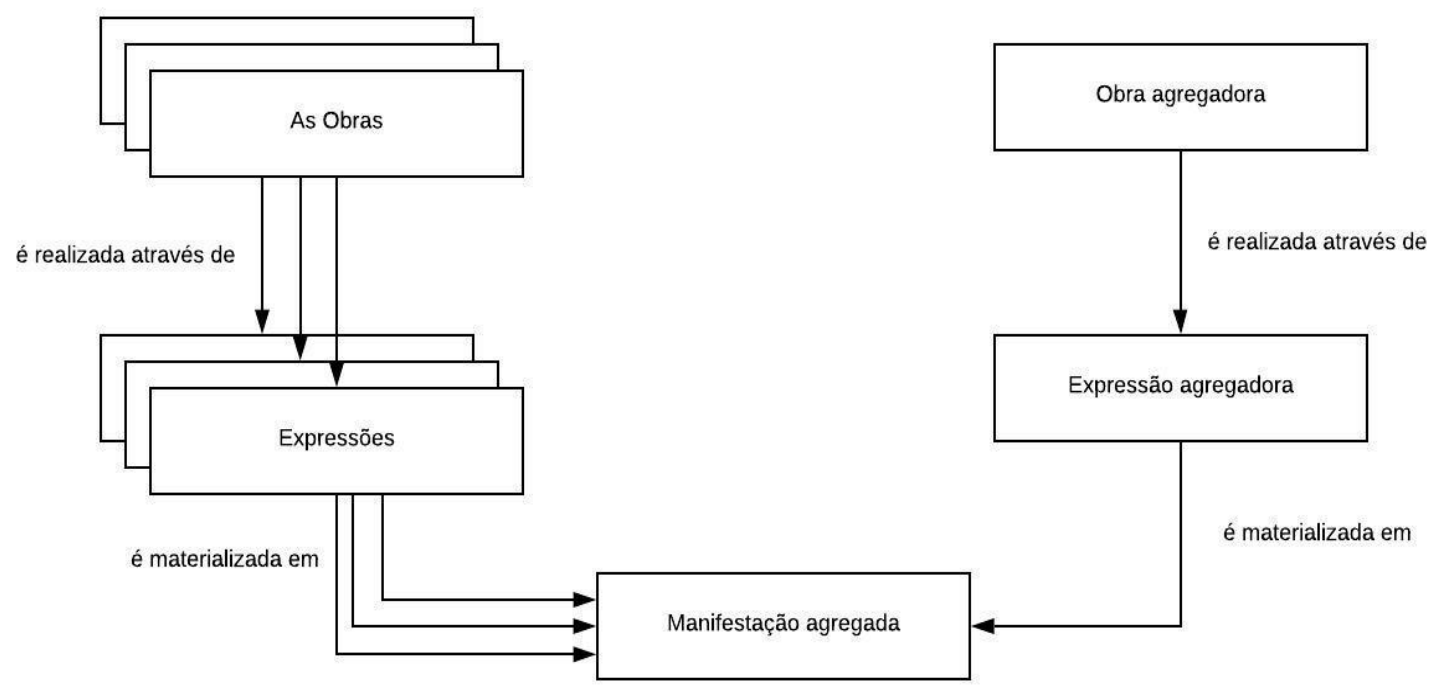

Fonte: Riva, LeBouef e Žumer (2017, p. 97).

A postulação de Jones (2018) refere-se à abordagem do IFLA LRM que trata as publicações seriadas como um caso específico de obra agregada, que implica em "[...] construções complexas que combinam relações de todo/parte e relações de agregação". (RIVA; LEBOEUF; ŽUMER, 2017, p. 97). 
Abrahamse (2019, p. 12-13, tradução nossa), sobre o tratamento do IFLA LRM de autores fictícios, que restringe autoria às pessoas reais, e o que pode não ser "[...] um apropriado uso de esforço e tempo de catalogadores", aponta que:

[...] essa insistência em restringir autoria a agentes humanos reais parece a certos catalogadores, como uma desnecessária e talvez, indesejada intrusão em prática estabelecida. Há uma preocupação de que, caso as bibliotecas adotem o LRM (como operacionalizado através do RDA revisado), os catalogadores precisarão revisar essa prática, e cessar de atribuir a função de autoria àqueles "autores" que não podem ser claramente traçados a pessoas reais. Isso pode nos exigir olhar retrospectivamente a registros já existentes e os trazer à concordância com o novo entendimento, de o que pode e o que não pode ser tratado como propriamente um autor.

Concernente a autores fictícios, o IFLA LRM, diferentemente do FRBR e FRAD, é explícito na definição da entidade pessoa, que diz respeito a "um ser humano individual" (RIVA; LEBOEUF; ŽUMER, 2017). Acrescentando ainda, em notas de escopo que:

A entidade pessoa é restrita a pessoas reais que vivem ou presumese que tenham vivido. Não é necessária uma prova estrita da existência de uma pessoa, desde que haja uma aceitação geral de sua provável historicidade. No entanto, figuras geralmente consideradas fictícias (por exemplo, Caco o Sapo), literárias (por exemplo, Miss Jane Marple) ou puramente lendárias (por exemplo, o mago Merlin) não são instâncias da entidade pessoa (RIVA; LEBOEUF; ŽUMER, 2017, p. 30).

Entretanto, o modelo não ignora identidades bibliográficas (ou personas), apesar de privá-las de autoria, e prescreve que

[...] uma identidade bibliográfica é um agrupamento de nomens utilizado por uma pessoa no mesmo contexto ou contextos bibliograficamente significativos. Os diferentes tipos de contexto de uso acionam o reconhecimento e o consequente tratamento específico de distintas identidades bibliográficas dependendo das normas de catalogação ou do sistema de organização do conhecimento. Por exemplo, vários pseudônimos para a mesma pessoa podem exigir vários pontos de acesso preferidos nas normas de catalogação, mas apenas um único número de classificação (RIVA; LEBOEUF; ŽUMER, 2017, p. 92).

Dessa maneira, uma identidade bibliográfica deve ser representada por um nomen da entidade pessoa. Um exemplo seria: a entidade pessoa Fernando 
Pessoa possui vários nomens, referindo-se a seus heterônimos Alberto Caeiro, Ricardo Reis, Álvaro de Campos e Bernardo Soares; esses nomens são agrupados juntamente com outros nomens, referentes a variados identificadores e diferentes línguas, formando um cluster de nomens que dizem respeito ao mesmo contexto de uso. O mesmo procedimento ocorre com pseudônimos, como no caso do autor e matemático Charles Lutwidge Dodgson, conhecido pelo seu pseudônimo Lewis Carroll.

No caso de personagens e lugares lendários ou ficcionais (por exemplo, Merlin, Sherlock Holmes, Sísifo, Gandalf, Terra Média, Atlantis, Avalon etc.), não são considerados, respectivamente, identidades bibliográficas ou instâncias da entidade Lugar. Em ambos os casos, o IFLA LRM considera-os como instâncias da entidade Res, conforme os exemplos da tabela 4.2 de seu documento final (RIVA; LEBOEUF; ŽUMER, 2017).

Ao aplicar os mecanismos de extensão do modelo, Padron, Cruz e Silva (2020) encontraram dificuldades concernentes ao "posicionamento de conceitos de domínio específico nas entidades OEMI", além da "falta de expressividade" da linguagem EER, que se utiliza de relacionamentos entre apenas duas entidades.

Porém, como Žumer (2018, p. 312, tradução nossa) assinala, a estrutura hierárquica de classes e subclasses provida pela modelagem EER, é

[...] um poderoso mecanismo que possibilita uma considerável simplificação: atributos e relacionamentos, declarados em um nível superior, são herdados por todas as subclasses e não necessitam de repetição em níveis mais baixos.

Sobre as publicações que tratam da implementação do IFLA LRM pelo RDA, Adamich $(2017,2018)$ declara que tal ação favorece o uso do padrão em cenários da catalogação descritiva baseados em Linked Data e na Web Semântica. Sendo assim, o autor destaca o Quádruplo Caminho (do inglês, the 4-Fold Path) do RDA 3R Project, consequência da implementação do modelo, que consiste em diretrizes que indicam como uma entidade deve ser representada e quais os métodos devem ser utilizados. Adamich $(2017,2018)$ 
faz a inferência de que, conforme o crescimento da inserção do Linked Data no universo bibliográfico, o Quádruplo Caminho certamente expandirá em estrutura e escopo.

No que tange aos métodos supracitados, Adamich (2018, p. 17, tradução nossa), os expõe da seguinte maneira:

1. Descrição Desestruturada - registrada como nota e a única opção a ser utilizada em caso de transcrição;

2. Descrição Estruturada - codificada utilizando-se de um esquema reconhecido (ex: pontuação ISBD, sintaxe de declaração de autoridade);

3. Identificador - códigos universais como o ISBN, ISSN, ISAN, ISNI, ORCID etc.

4. IRI (Internationalized Resource Identifier) - link da web persistente e acionável, atribuído unicamente à entidade.

O Quádruplo Caminho deriva da postulação do IFLA LRM de duas maneiras de representação de um atributo em uma implementação real. Essas duas maneiras, conforme está escrito no documento do modelo, são:

- um atributo pode ser representado como um mero literal (uma string, um número ...): é isso que o OWL (Web Ontology Language) considera como "propriedades de tipo de dados";

- um atributo pode ser representado como um Uniform Resource Identifier (URI) apontando para uma fonte externa (um documento referencial ou normativo de qualquer tipo, como um arquivo de autoridade ou uma lista de valores codificados); nesse caso, poderia ter sido modelado como um relacionamento, e não como um mero atributo, mas o modelo deve permanecer independente do modo como deve ser implementado: é isso que a OWL considera como "propriedades do objeto" (RIVA; LEBOEUF; ŽUMER, 2017, p. 11-12).

El-Sherbini (2016) destaca no IFLA LRM, justamente essa possibilidade de representação de um atributo por meio de um URI ligado a recursos externos. Ademais, Goldberga et al. (2018), em um artigo sobre a coleção Linked Data Rainis And ASpazija (RunA), listam como ponto forte do RDA, a sua compatibilidade com o IFLA LRM, que possibilita o suporte de hierarquia de entidades. Ademais, como resultado do RDA Toolkit Restructure and Redesign (3R) Project, iniciado em 2016 e finalizado em dezembro de 
2020, o RDA tornou-se oficialmente compatível com o IFLA LRM, tomando-o como base estrutural (RDA Toolkit, 2020).

Gemberling (2016) aborda o FRSAD e o IFLA LRM a partir de uma perspectiva argumentativa filosófica, a partir do antagonismo entre realismo e nominalismo. É argumentada e defendida a perspectiva realista ${ }^{6}$ adotada pelo FRSAD, que permite a entidade Thema (conceito do assunto) ser representada por diferentes Nomens (termo de assunto), "como "tree" em inglês e "arbol" em espanhol”. Ao discutir o IFLA LRM, Gemberling (2016, p. 142, tradução nossa) admite que o modelo é construído respaldado nessa visão realista, porém discorda da substituição da entidade Thema pela Res.

[...] enquanto isso aparenta ser um ganho em simplicidade, na verdade é uma perda de discernimento. O problema é que enquanto qualquer coisa (qualquer Res) pode ser o referente de um signo, ele só é realmente um se há um interpretante para tanto. Retendo a entidade Thema nos ajuda a reconhecer que o signo potencial das coisas (seu "Thema-ness") é distinto da sua mera existência. ${ }^{7}$

Essa abordagem filosófica de Gemberling (2016), vai ao encontro dos apontamentos de Maclennan e Walicka (2020), que dizem respeito a problemas concernentes à dificuldade de compreensão das abstrações do IFLA LRM por parte da comunidade catalográfica. Como Gemberling (2016) postula, essa abordagem oferece um embasamento para a justificação de algumas tomadas de decisão dos modelos, o que por sua vez pode facilitar a compreensão de tais "abstrações", tornando essa uma abordagem relevante para futuras pesquisas sobre os modelos conceituais.

Dessa maneira, o Quadro 4 sintetiza as vantagens e desvantagens abordadas na literatura científica.

Quadro 4 - Sumarização das vantagens e das desvantagens do IFLA LRM

\begin{tabular}{|c|c|}
\hline Vantagens & Desvantagens \\
\hline - Modelo abstrato com estrutura lógica do \\
$\begin{array}{c}\text { universo bibliográfico. } \\
\text { - Perda de granularidade, poder funcional e } \\
\text { explanatório em relação aos modelos da } \\
\text { Família FR. }\end{array}$ \\
\hline
\end{tabular}




\begin{tabular}{|c|c|}
\hline $\begin{array}{l}\text { - Consistente e simples no agrupamento } \\
\text { de recursos intelectuais. }\end{array}$ & $\begin{array}{c}\text { - Restrição de autoria à agentes humanos } \\
\text { reais. }\end{array}$ \\
\hline $\begin{array}{l}\text { - Tarefas dos usuários que abrangem } \\
\text { casos de busca de informação realizados } \\
\text { em qualquer cenário e plataforma. }\end{array}$ & $\begin{array}{l}\text { - Falta de expressividade da linguagem } \\
\text { EER. }\end{array}$ \\
\hline - Alinhado com a visão do usuário. & $\begin{array}{l}\text { - Mais distante de implementação do que } \\
\text { outros modelos, devido à seu alto nível de } \\
\text { abstração. }\end{array}$ \\
\hline $\begin{array}{c}\text { - Alinhado com a Web Semântica e } \\
\text { Linked Data }\end{array}$ & - Abstrações de difícil compreensão. \\
\hline $\begin{array}{l}\text { - Possibilita a representação de um } \\
\text { atributo através de um URI. }\end{array}$ & $\begin{array}{c}\text { - Apresenta uma abordagem da ciência dos } \\
\text { dados ao exercício de catalogação } \\
\text { descritiva. }\end{array}$ \\
\hline - Sustenta a hierarquia das entidades. & - \\
\hline
\end{tabular}

Fonte: Elaborado pelos autores.

As vantagens e as desvantagens da adoção do modelo IFLA LRM em sua totalidade mostram-se niveladas nos artigos analisados e predomina uma abordagem mais no campo conceitual, de maneira que poucas partem de alguma aplicação prática. Assim, as questões analisadas e discutidas dirigem-se não ao "produto final" do modelo, mas às tomadas de decisões na escolha de diretrizes para sua elaboração.

\section{Conclusão}

A partir da realização de uma Revisão Sistemática da Literatura, essa pesquisa teve como objetivo apresentar o estado da arte do modelo IFLA LRM na literatura científica disponível, principalmente por meio de seus casos de uso, das vantagens e das desvantagens.

Foram recuperados 168 artigos por meio da busca nas bases de dados especificadas no protocolo de Revisão Sistemática da Literatura. Destes, após a aplicação dos critérios de inclusão e de exclusão foram aceitos 39 documentos, sendo que apenas 6 incluíam casos de uso.

Os casos de uso apresentam resultados positivos e significativos ao IFLA LRM, e justificam grande parte das diretrizes tomadas na consolidação da 
Família FR, que em outros artigos apareceram sob o espectro conceitual e tomadas como desvantagens.

Em suma, os casos de uso do IFLA LRM apresentam resultados positivos, que por sua vez indicam um caminho fértil e promissor para a criação de modelos de extensão direcionados a diferentes áreas e tipos de dados, fator essencial para seu desenvolvimento e inserção na prática catalográfica.

Outrossim, a implementação do IFLA LRM pelo RDA é tomada como uma iminente evolução do padrão de catalogação, e delineiam-se futuras discussões sobre seu tratamento de autores fictícios e obras seriadas, uma vez que, nessas questões, o modelo rompe com a tradição catalográfica.

Esse estudo, apesar de não conclusivo, se apresenta como um caminho inicial, para que a comunidade de bibliotecários, possa refletir a natureza eminentemente tecnológica que está imbricada na prática catalográfica, além de contribuir com uma análise sistematizada da literatura científica, que auxilie na continuidade de pesquisas emergentes e potenciais na Catalogação Descritiva.

\section{Financiamento}

Agradecemos ao Conselho Nacional de Desenvolvimento Científico e Tecnológico $(\mathrm{CNPq})$ pelo apoio financeiro à pesquisa.

\section{Referências}

AALBERG, Trond; TAALERÅS, Kim; MASSEY, David. The impact of new bibliographic models on the search experience. Information Research, Suécia, v. 24, n. 4, dez. 2019.

ABRAHAMSE, Ben. What if superman wrote a memoir? the curious case of fictional authors under LRM and the new RDA. Technicalities, [S. 1.], v. 39, n. 5, p. 12-14, set./out. 2019.

ADAMICH, Tom. RDA toolkit relaunch. Technicalities, [s. 1.], v. 38, n. 3, p. 16-19, maio/jun. 2018.

ADAMICH, Tom. FRBR models and RDA updates. Technicalities, [s. 1.], v. 37, n. 5, p. 14-17, set./out. 2017. 
ARASTOOPOOR, Sholeh. Users' perception of navigating bibliographic families from IFLA-LRM perspective. Library Hi Tech, [s. 1.], Vol. ahead-ofprint, No. ahead-of-print., dez. 2020.

BENELLI, Sabina. Italian Cataloguing Rules for Unpublished Music Resources. Fontes Artis Musicae, [s. 1.], v. 67, n. 4, p. 319-330, out./dez. 2020.

COYLE, Karen. FRBR, before and after: a look at our bibliographic models. Chicago: American Library Association, 2016.

DUNSIRE, Gordon. FRITZ, Deborah. FRITZ, Richard. Instructions, interfaces, and interoperable data: the RIMMF experience with RDA revisited. Cataloging \& Classification Quarterly, [s. 1.], v. 58, n. 1, p. 44-58, dez. 2019..

EL-SHERBINI, Magda. RDA implementation and the emergence of BIBFRAME. JLIS.it., [Italy], v. 9, n. 1, p. 66-82, jan. 2018.

FREDERICK, Donna Ellen. Information seeking in the age of the data deluge. Library Hi Tech News, [s. 1.], v. 36, n. 2, p. 6-10, abr. $2019 .$.

GALVÃO, Maria Cristina Barbosa; RICARTE, Ivan Luiz Marques. Revisão sistemática da literatura: conceituação, produção e publicação. LOGEION: Filosofia da Informação, Rio de Janeiro, v. 6, n. 1. p. 57-73, set. 2019/fev. 2020.

GEMBERLING, Ted. FRSAD, semiotics and FRBR-LRM. Cataloging \& Classification Quartely, [s. 1.], v. 54, n. 2, p. 136-144, jan.2016..

GOLDBERGA, Anita; KREISLERE, Maira; RAŠMANE, Anita; STŪMANE, Aiva; SALNA, Edgars. Identification of entities in the linked data collection "Rainis and Aspazija" (RunA). JLIS.it., [Italy], v. 9, n. 1, p. 83-106, jan. 2018.

HIDER, Philip. A critique of FRBR user tasks and their modifications.

Cataloguing \& Classification Quartely, [s. 1.], v. 55, n. 2, p. 55-74, dez. 2016.

IFLA. International Federation of Library Associations and Institutions.

Declaração dos Princípios Internacionais de Catalogação. 2016.

JESUS, Ananda Fernanda de; CASTRO, Fabiano Ferreira de. Dados bibliográficos para o linked data: uma revisão sistemática de literatura.

Brazilian Journal of Information Studies: Research Trends, Marília, v. 13, n.1, p. 45-55, 2019.

JONES, Edgar. The Evolution of the serial work, the FRBR conceptual model, and RDA. Cataloging \& Classification Quarterly. [s. 1.], v. 56, n. 2/3, p. 128145, nov. 2017. 
MACLENNAN, Alan.; WALICKA, Agnieszka. An investigation into cataloguers' experience with RDA. Journal of librarianship and information science. [s. 1.]. v. 52, n. 2, p. 464-475, jan. 2017.

MARCONDES, Carlos H. Towards a vocabulary to implement culturally relevant relationships between digital collections in heritage institutions. Knowledge Organization. [s. 1.], v. 47, n. 2, p. 122-137, jan. 2020.

PADRON, Marcos Fragomeni; CRUZ, Fernando William; SILVA, Juliana Rocha de Faria. Extending the IFLA Library Reference Model for a Brazilian popular music digital library. International Journal on Digital Libraries. [s. 1.], v. 21, p. 289-306, jan. 2020.

PENNINGTON, Diane Rasmussen; CAGNAZZO, Laura. Connecting the silos: implementations and perceptions of linked data across european libraries. Journal of Documentation, [s. 1.], v. 75, n. 3, p. 643-666, dez. 2018.

RDA Toolkit. What you should know about the December Switchover. 2020.

RIVA, Pat; LE BOUEF, Patrick; ŽUMER, Maja. IFLA Library Reference Model: um modelo conceitual para a Informação Bibliográfica, [s. 1.]: Internacional Federation of Library Associations and Institutions, 2017.

SIMIONATO, Ana Carolina. Família FR, BibFrame, e linked data: integração de dados bibliográficos. In: Encontro de Representação Documental - EnReDo, 1., 2017. Anais [...]. São Carlos: Universidade Federal de São Carlos, 2017.

SPROCHI, Amanda. Where are we headed? Resource Description Access, Bibliographic Framework, and the Functional Requirements for Bibliographic Record Library Reference Model. International Information \& Library Review. [s. 1.], v. 48, n. 2, p. 129-136, jun. 2016.

STRADER, C. R. From User tasks to user services: placing the functional requirements for bibliographic records models into a larger framework. Technical Services Quarterly. [s. 1.], v. 34, n. 4, p. 347-368, ago. 2017.

TANIGUCHI, S. Mapping and merging of IFLA Library Reference Model and BIBFRAME 2.0. Cataloging \& Classification Quarterly. [s. 1.], v. 56, n. 5/6, p. 427-454, dez. 2018.

ŽUMER, Maja. IFLA Library Reference Model (IFLA LRM): harmonisation of the FRBR Family. Knowledge Organization. [S. 1.], v. 45, n. 4, p. 310-318, maio 2018. 


\title{
The impact of the IFLA Library Reference Model on cataloging practice: use cases, advantages and disadvantages
}

\begin{abstract}
The IFLA Library Reference Model (IFLA LRM) is a high-level conceptual model that arises from the need to consolidate the three models of the FR Family, the demands of the current technological context and compatibility with the best practices of Linked Data. The IFLA LRM treats bibliographic data in a broad sense, explaining the general principles that govern the logical structure of bibliographic information, as the basis for formulating cataloging rules and implementations of bibliographic systems. Thus, this research aims to identify the state of the art of the IFLA LRM model, based on the available scientific literature. As a research method, the Systematic Literature Review (RSL) was adopted in order to build the theoretical framework. For the analysis of the documents, the following categories were established: (I) focus of the documents; (ii) IFLA LRM concept; (iii) relationship between IFLA LRM and Linked Data; (iv) advantages and disadvantages of the model; (v) IFLA LRM use cases. As a result, the state of the art on the IFLA LRM model is presented, as well as the research trends and the impact of the model on cataloging practice, as presented in the identification of use cases. It is concluded that the IFLA LRM model presents itself as an object of investigation of capital importance, since it reflects the changes in Descriptive Cataloging and the new directions of the area in the digital context, in the adequacy, sharing and reuse of bibliographic data in the scenery of Semantic Web and Linked Data.
\end{abstract}

Keywords: Descriptive cataloging; Conceptual models; Bibliographic data; Linked Data; IFLA LRM

Recebido: 13/04/2021

Aceito: 02/07/2021

\section{Declaração de autoria}

Concepção e elaboração do estudo: Oliveira e Castro

Coleta de dados: Oliveira

Análise e interpretação de dados: Oliveira, Castro e Jesus

Redação: Oliveira e Castro

Revisão crítica do manuscrito: Oliveira, Castro e Jesus 


\section{Como citar}

OLIVEIRA, Rhuan Henrique Alves de; CASTRO, Fabiano Ferreira de; JESUS, Ananda Fernanda de. O impacto do modelo IFLA Library Reference Model na prática catalográfica: casos de uso, vantagens e desvantagens. Em Questão, Porto Alegre, v. 27; n. 4, p. 359-386, 2021 .Doi: http://dx.doi.org/10.19132/1808-5245274.359-386

1 "Universo relacionado às coleções de bibliotecas, podendo incluir também coleções de arquivos e museus". (IFLA, 2016, p. 18).

${ }^{2}$ Um modelo conceitual de alto nível possui uma abordagem ampla e geral do domínio que se propõe a estruturar, de tal maneira que não se faz suficiente para uma aplicação direta.

${ }^{3}$ Entidades basilares do IFLA LRM: Obra, Expressão, Manifestação e Item.

4 "Cada elemento do modelo é numerado para referência inequívoca. A convenção de numeração adotada é o prefixo "LRM-", uma letra correspondente ao tipo de elemento (E = entidade; $\mathrm{A}=$ atributo; $\mathrm{R}$ = relacionamento) e um número sequencial”. (RIVA; LEBOEUF; ŽUMER, 2017).

${ }^{5}$ A Web Ontology Language (OWL) é uma linguagem da Web Semântica para a construção de ontologias.

6 “A perspectiva realista, condensada por Platão, assim como pelo oponente de Ockham, John Duns Scotus (ca. 1266-1308), enfatiza a correspondência entre conceitos gerais com verdadeiras generalizações sobre o mundo". (GEMBERLING, 2016, p. 137, tradução nossa).

${ }^{7}$ Referente, Signo e Interpretante são elementos da Semiótica de Peirce dos quais dependem a funcionalidade de qualquer Signo. (GEMBERLING, 2016, tradução nossa). 